\title{
Ireland's potential MDR-TB and XDR-TB problem
}

\author{
M. B. O'Connor
}

Received: 30 April 2009/Accepted: 28 September 2010/Published online: 10 October 2010

(C) Royal Academy of Medicine in Ireland 2010

\section{Dear Editor,}

Multi-drug-resistant tuberculosis (MDR-TB) is defined as TB that is resistant at least to isoniazid (INH) and rifampicin (RMP), the two most powerful first-line anti-TB drugs. Isolates that are multiply resistant to any other combination of anti-TB drugs but not to INH and RMP are not classed as MDR-TB. MDR-TB mostly develops in the course of the treatment of fully sensitive TB and this is the result of patients missing doses, doctors giving inappropriate treatment, or patients failing to complete a course of treatment. It is unusual for MDR-TB to spread from person to person, except in the context of HIV or immune suppression. This compares to extensively drugresistant tuberculosis (XDR-TB) which is defined as TB that has developed resistance to at least RMP and INH, as well as to any member of the quinolone family and at least one second-line anti-TB injectable drugs: kanamycin, capreomycin, or amikacin. XDR-TB is felt to have emerged from the mismanagement of MDR-TB and can spread from one person to another.

MDR-TB strains appear to be less virulent and less transmissible, and outbreaks tend to occur in people with weakened immune systems (e.g. HIV) [1]. Outbreaks among immuno-competent healthy people do occur, but are uncommon [2]. A 1997 survey of 35 countries found rates above $2 \%$ in about a third of the countries surveyed. The highest rates were in the former USSR, the Baltic States, Argentina, India and China, and were associated with poor or failing national tuberculosis control programs. Unfortunately, the true scale of XDR-TB is unknown as many countries lack the necessary equipment and capacity to accurately diagnose it. It is estimated, however, that there

M. B. O’Connor ( $\square)$

Department of Medicine, South Infirmary-Victoria

University Hospital, Cork, Ireland

e-mail: mortimeroconnor@gmail.com are around 40,000 cases per year. As of June 2008, 49 countries have confirmed cases of XDR-TB [2].

The works of Friedland GH, Uzdaviniene V, and Podlekareva D which were presented at the 5th European Conference on Clinical and Social Research on AIDS and Drugs, in Lithuania, highlight the extent of the global MDR-TB/ XDR-TB problem. Friedland GH has highlighted the existence of resistant TB in the US and Africa, while Uzdaviniene V, and Podlekareva D highlighted the Eastern European problem, an area with a high HIV incidence in the setting of intravenous drug use (IDU). Of interest, all research showed a high association with the presence of active resistant TB and the presence of HIV and drug abuse. Ultimately, all of these studies recommended that in cases of resistant TB we should be testing for HIV and giving support from a drug addiction perspective.

From an Irish context, these studies have an impact. In a time of economic recession, concerns regarding medication adherence are to the fore, as is the case with substance abuse, and poor healthcare seeking behavior. Combining these with the migration knowledge that Ireland has large inward migration from Africa and Eastern Europe, we need to be aware of potential inward migration of MDR-TB and XDR-TB. We also should be aware of inward migration of HIV and a drug use/abuse culture. Ultimately, we should consider testing all MDR-TB and XDR-TB patients, in Ireland, for HIV and give appropriate drug abuse/addiction support where required. All of these are best accomplished by comprehensive, collaborative and integrated services.

\section{References}

1. Keshavjee S, Gelmanova IY, Farmer PE et al (2008) Treatment of extensively drug-resistant tuberculosis in Tomsk, Russia: a retrospective cohort study. Lancet 372(9647):1403-1409

2. World Health Organization (2008) Countries with XDR-TB confirmed cases as of June 2008. World Health Organization, Geneva 